\title{
Smart Home System Implementation Based on ARM Microprocessor
}

\author{
Dan $\mathrm{Li}$ \\ College of Electrical and Information Engineering, Jilin Agricultural Science and Technology \\ University, Jilin City, China \\ 653301981@qq.com
}

\begin{abstract}
Keywords: RFID technology; Smart home; ARM microprocessor; Wireless communication
\end{abstract}
\begin{abstract}
This paper describes a kind of smart home control system. The system can intelligently adjust the indoor environment, according to the temperature and humidity, the light intensity, the combustible gas levels and other parameters. The smart home control system can intelligently control household appliances in order to achieve energy saving purpose. It uses embedded microprocessor as control unit, and uses RFID technology for household staff identity recognition. The smart home control system uses sensor system to get indoor environment parameters. Through the real-time measurement and control, it helps people to achieve comfortable, intelligent household environment.
\end{abstract}

\section{Introduction}

The use of household appliances is an essential part of people's busy lives. But many people often forgot to turn off the power, or forget to turn off the appliances. It is not only a waste of energy but also security risk. For example, while no one passes by the public staircase light, the light is always on, this is a waste of electricity. We can make the street lamp brighten while someone passes through. While people walk past, the street lamp lights out again, it is not only to save energy, but also does not affect the normal use. [1]

Life theme of energy conservation and environmental protection, makes the smart home system more noticeable. The difference between the smart home system and the traditional household system is that the smart home system gives the family all items with "wisdom", and enables them to "spontaneously", "actively" to communicate with people and realize the information interaction. At first, RFID technology can enable smart home systems automatically recognize, perceive household appliances, systems and intelligent management, so as to achieve energy-saving automatic control purposes. [2]

\section{The Design of Overall System}

This system uses a microprocessor as the control core and uses RFID radio frequency technology as a means of identification. This system the need for personnel to carry cards in and out of the room door, and microprocessor read the door card number and information to identify the guest in this room. Intelligent lighting management system is an important part of the system. After people leave the room, the microprocessor control modules can determine whether there are somebody in room, if no one, will cut off the power supply conduct electricity; when to enter the room, the room again. Intelligent lighting management system in addition to the traditional functions, the robot also can sense the human movement and the surrounding environment such as illumination; in order to automatically control the light switch and the dimming, do " people come to light, people go the lights " lights went out. [3]Intelligent lighting control system can simulate according to the need of the change of the natural sunlight, the user as long as the light touch switch can feel or in the hands of the remote control from summer to winter, from spring to autumn to simulate seasonal change, even can simulate different times of day.

As shown in Fig. 1, the system applies a variety of sensors to collect indoor physical parameters, then through a wide range of information to judge people, environment conditions. 


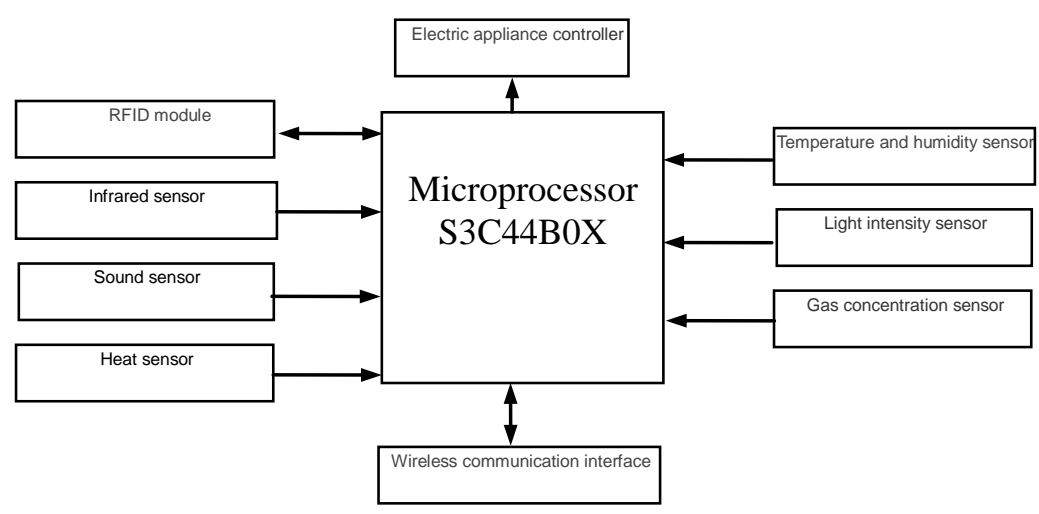

Figure 1. System composition block diagram

\section{The Design of Indoor Personnel Detection System}

The microprocessor uses infrared sensors, sound sensors, and heat sensors to collect information for analyzing indoor physical parameters. Infrared sensor is the first layer detection system. When someone enters the room infrared sensor can sense. Sound sensor is the second layer detection system, and heat sensor is the third layer detection system. After comprehensive analysis, the microprocessor gets the number of indoor person and then provides household appliances drive circuit with power supply. When people walk out from the room, infrared sensor is firstly judging whether someone is in the room, and then the sound sensor, the final is heat sensor. Only when the three kinds of sensors are determined that no one else is in the room, the system shut down the power. When someone enters the room, RFID system will test whether there is any room card. RFID will test your room card whether meet the requirements. If there is a room card and meets the requirements, the indoor personnel detection system will run; otherwise, the photograph will be made for anti-theft function. [4]

\section{The Design of Indoor Environment Factor Detection System}

AM2303 humidity-sensitive capacitance digital temperature and humidity module is the temperature and humidity sensor which has already calibrated digital composite signal output. It uses digital acquisition technology and the temperature and humidity sensor technology to ensure that the product has high reliability and excellent long-term stability. [5]The sensor includes a capacitive humidity sensitive element and a high precision temperature measurement element, which are connected to a high performance 8-bit microprocessor. Therefore, the product has the advantages such as excellent quality, fast response, strong anti-interference ability, high performance price ratio.

After power on, AM2303 measures the environment temperature and humidity parameters, and records the data, then automatically turns to dormant state. The AM2303 SDA data line is raised and maintained high level by a pull-up resistor. The SDA pin is input state, ready for detecting external signals. The microprocessor I/O pin is set to output, and output low voltage signal. Then the processor pin is set as the input state. After the host releases the bus, AM2303 sends a response signal, which is followed by the data bus SDA continuous outputting. AM2303 measure again environment temperature and humidity, and record data. [6]

\section{The Design of Wireless Communication Interface Module}

The function of the wireless communication interface module is to complete the communication between indoor control system and a handheld device, which completes the environment control with wireless operations. Chip nRF905 is a single-chip radio transceiver chip, which works on 433/868/915 MHZ ISM frequency band. The Shock Burst function of nRF905 can easily achieve high data rate, without the need of a expensive high-speed microprocessor to process data and deal 
with clock recovery. [7] In the application, nRF905 Shock Burst function can reduce the average current consumption. In the receive mode of the Shock Burst function, when a valid address and a valid data was received, address matching pin (AM) and data ready pin (DR) will promptly notify the microprocessor. [8]In the transmission mode of Shock Burst function, it will automatically generate sync signal and CRC check code, DR notifies the microprocessor that data transmission has been completed. Wireless communication interface chip nRF905 circuit principle diagram is shown in Fig. 2.

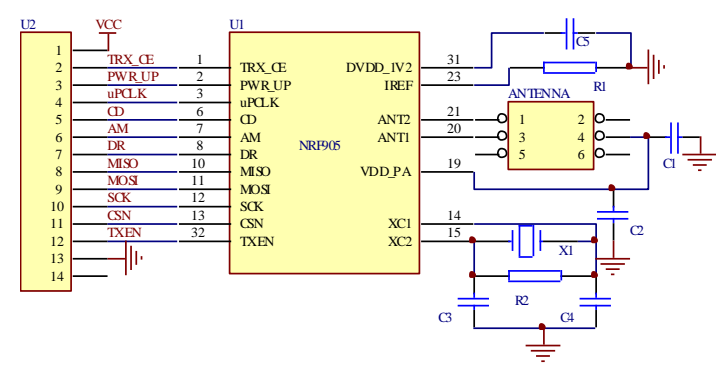

Figure 2. nRF905 circuit principle diagram

\section{The Design of RFID Module}

The Hardware Design of RFID Module. This system adopts the ISO/IEC 14443 protocol Mifare one S50 format RFID tag and read/write device. The smart home system users wear RFID tags, of which the electrical part only consists of an antenna and ASIC. Electronic tag antenna is only a few groups of coil winding, very suitable for packaging into ISO electronic tag. The ASIC electronic tag consists of a high speed (106 KB baud rate) RF interface, a control unit and an EEPROM. Transmitting or receiving data is stored in EEPROM of Mifare One tag. EEPROM is divided into 16 sectors, and each sector is made up of four sectors. Sector 0 Blocks 0 (the absolute address block 0 ) is used to store vendor code, has been solidified, cannot be changed. Each sector's block 0, block 1, block 2 can be used to store data. Data blocks can be used for two fields: one field is general data, which you can carry out read and write operations; another field is data value, which you can initialize values, add value, read value operations. Each sector block 3 is control block, including the password A, access control, password B. [9]

As shown in Fig. 3, the system processor communicates with RFID read-write module by UART interface, and completes the user identity recognition. The microprocessor sends commands to the RFID read-write module chip through the UART interface. When there is RFID card within the effective scope of work, the card will reply card types; establish contact with the read/write module card. [10]



Figure 3. CY-14443A module interface schematic diagram 
The Software Design of RFID Read-write Module. The array assignment core code of RFID cmdsend command frame is as follows:

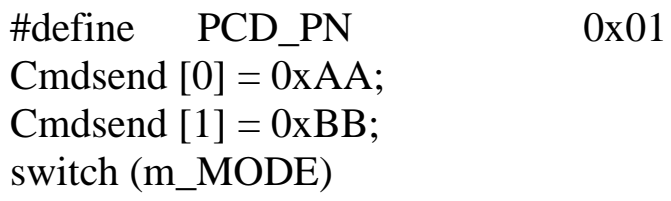

case 6: //The 6th command word in the command table is to set automatic finding card mode. $\ldots .$.

\section{Conclusion}

The household circuit system, introduced in this paper, takes the microprocessor as the core, RFID radio frequency identification technology is adopted to improve the information collection, automatically and accurately judgment and more intelligently, more accurately control the power supply circuit, to achieve the purpose of energy-efficient appliances. In the laboratory environment, the system achieves the purpose of work stability, good performance, electricity energy saving effect, and it achieves the practical purpose of energy saving and emission reduction.

\section{Acknowledgements}

This paper is supported by Key Subject Cultivation Project of Jilin Agricultural Science and Technology University (NO.2015X093).

This paper is supported by Youth Fund Project of Jilin Agricultural Science and Technology University (NO.2015224).

This paper is supported by Higher Education Scientific Research Project of Jilin Provincial Institute for Higher Education (JGJX2015C90) and supported by Jilin Province education science 12th Five-Year foundation (GH14335).

\section{References}

[1] Yi Wang: Design of the Smart Home Control System Based on Internet of Things, Software Engineering and Applications, Vol. 3(2014)No. 2, p.23.(in Chinese).

[2] Ren Gao: Design and Implementation of Smart Home Based on Wireless Sensor Networks, Hans Journal of Wireless Communications, Vol. 2(2012) No.4, p. 85(in Chinese).

[3] Lin Gao: Design of Smart Home System Based on ZigBee Technology and R\&D for Application, Energy and Power Engineering, Vol. 8(2016) No. 1, p. 13.

[4] Ameena Saad al-sumaiti:Smart Home Activities: A Literature Review, Electric Power Components and Systems, Vol.42(2014)No.3,p.294

[5] Iram Fatima: Analysis and effects of smart home dataset characteristics for daily life activity recognition, The Journal of Supercomputing, Vol.66(2013)No.2,p.760

[6] Jasmina Mandic-Lukic: Impact of stand-by energy losses in electronic devices on smart network performance, Thermal Science,Vol.16(2012)No.4,p.1239

[7] Sang Oh Park: An object-based middleware supporting efficient interoperability on a smart home network, Multimedia Tools and Applications,Vol.63(2013)No.1,p.227 
[8] Habib F. Rashvand: Distributed Sensor Systems (John Wiley \& Sons, Ltd, UK 2012 ), p.10.

[9] Drubin: More than Half a Billion Wireless Smart Home Monitoring Devices to be Installed Worldwide by 2018, Microwave Journal, Vol.57(2014)No.5,p.81

[10]P.C. Tseng: The design of a single-chip tool monitoring system for on-line turning operation, The International Journal of Advanced Manufacturing Technology, Vol. 24(2004) No. 5, p.404. 